\title{
182. On Moduli of Open Holomorphic Maps of Compact Complex Manifolds
}

\author{
By Makoto NAMBA \\ Tohoku University \\ (Comm. by Kunihiko KodaIRA, M. J. A., Dec. 12, 1974)
}

1. Let $V$ and $W$ be connected compact complex manifolds. According to Douady [1], the set $H(V, W)$ of all holomorphic maps of $V$ into $W$ admits an analytic space ${ }^{*)}$ structure whose underlying topology is the compact-open topology. We denote by $O(V, W)$ the set of all open holomorphic maps of $V$ onto $W$. Then $O(V, W)$ is an open subvariety of $H(V, W)$. Let $\operatorname{Aut}(V)$ and $\operatorname{Aut}(W)$ be the automorphism groups of $V$ and $W$, respectively. It is well known that they are complex Lie groups. Now, $\operatorname{Aut}(W)$ and $\operatorname{Aut}(W) \times \operatorname{Aut}(V)$ act on $O(V, W)$ as follows:

$$
\begin{aligned}
& (b, f) \in \operatorname{Aut}(W) \times O(V, W) \longrightarrow b f \in O(V, W), \\
& (b, a, f) \in \operatorname{Aut}(W) \times \operatorname{Aut}(V) \times O(V, W) \longrightarrow b f a^{-1} \in O(V, W) .
\end{aligned}
$$

In this note, we state the following theorems. Details will be published elsewhere.

Theorem 1. The orbit space $O(V, W) /$ Aut $(W)$ admits an analytic space structure such that the canonical projection map

$$
\pi: O(V, W) \longrightarrow O(V, W) / \operatorname{Aut}(W)
$$

is holomorphic and is a principal fiber bundle with the structure group $\operatorname{Aut}(W)$.

Theorem 2. Assume that Aut $(V)$ is compact. Then the orbit space $O(V, W) /(\operatorname{Aut}(W) \times \operatorname{Aut}(V))$ with the quotient topology admits an analytic space structure such that (1) the canonical projection map

$$
\mu: O(V, W) \longrightarrow O(V, W) /(\operatorname{Aut}(W) \times \operatorname{Aut}(V))
$$

is holomorphic and such that (2) for any open subset $U$ of $O(V, W)$ and for any holomorphic map $F$ of $U$ into an analytic space $X$ which takes the same value at $(\operatorname{Aut}(W) \times \operatorname{Aut}(V)$ )-equivalent points, there is a holomorphic map $\hat{F}$ of $\mu(U)$ into $X$ with $\hat{F} \mu=F$.

Remark 1. The analytic space $O(V, W) /(\operatorname{Aut}(W) \times \operatorname{Aut}(V))$ in Theorem 2 is considered as the moduli space of open holomorphic maps of $V$ onto $W$.

Remark 2. Theorems 1 and 2 are proved by applying Holmann's works [2] and [3].

2. Aut $(V)$ acts on $O(V, W) / \operatorname{Aut}(W)$ as follows :

*) By an analytic space, we mean a reduced, Hausdorff, complex analytic space. 


$$
\begin{aligned}
& (a, \pi(f)) \in \operatorname{Aut}(V) \times(O(V, W) / \operatorname{Aut}(W)) \\
& \longrightarrow \pi\left(f a^{-1}\right) \in O(V, W) / \operatorname{Aut}(W) .
\end{aligned}
$$

Assume that Aut $(V)$ is compact. By Satz 20, [3], the orbit space $(O(V, W) / \operatorname{Aut}(W)) / \operatorname{Aut}(V)$ is an analytic space. We have

Proposition. Assume that Aut $(V)$ is compact. Then there is a canonical holomorphic isomorphism:

$O(V, W) /(\operatorname{Aut}(W) \times \operatorname{Aut}(V)) \cong(O(V, W) / \operatorname{Aut}(W)) / \operatorname{Aut}(V)$.

3. Let $V$ be a compact Riemann surface of genus $g \geqq 1$. Let $\boldsymbol{P}^{1}$ be the complex projective line. Then $O\left(V, \boldsymbol{P}^{1}\right)$ is the set of all non-constant algebraic functions on $V$. The analytic space $O\left(V, \boldsymbol{P}^{1}\right) /\left(\operatorname{Aut}\left(\boldsymbol{P}^{1}\right)\right.$ $\times \operatorname{Aut}(V))$ is considered as the moduli space of algebraic functions on $V$.

In particular, for a complex 1-torus $T, O\left(T, \boldsymbol{P}^{1}\right) /\left(\operatorname{Aut}\left(\boldsymbol{P}^{1}\right) \times \operatorname{Aut}(T)\right)$ is decomposed into the connected components as follows :

$$
O\left(T, \boldsymbol{P}^{1}\right) /\left(\operatorname{Aut}\left(\boldsymbol{P}^{1}\right) \times \operatorname{Aut}(T)\right)=M_{2} \cup M_{3} \cup \cdots,
$$

where each $M_{n}, n=2,3, \cdots$, is the moduli space of elliptic functions of order $n$ on $T$ and is an irreducible normal analytic space of dimension $2 n-4$. ( $M_{2}$ is one point.)

On the other hand, we can easily show that the orbit space $O\left(\boldsymbol{P}^{1}, \boldsymbol{P}^{1}\right) /\left(\operatorname{Aut}\left(\boldsymbol{P}^{1}\right) \times \operatorname{Aut}\left(\boldsymbol{P}^{1}\right)\right)$ is not Hausdorff.

\section{References}

[1] A. Douady: Le problème des modules pour les sous-espaces analytiques compacts d'un espace analytique donné. Ann. Inst. Fourier, Grenoble, 16, 1-95 (1966).

[2] H. Holmann: Quotienten komplexer Räume. Math. Ann., 142, 407-440 (1961).

[3] — : Komplexe Räume mit komplexen Transformationsgruppen. Math. Ann., 150, 327-360 (1963). 